\title{
Niveles de depresión y factores sociodemográficos en pacientes con insuficiencia renal crónica en tratamiento de hemodiálisis en Perú
}

\section{Levels of depression and sociodemographic factors in patients with chronic renal failure on hemodialysis treatment in Peru}

\author{
José Francisco Vallejos Saldarriaga ${ }^{12}$ \\ Eris Zoila Ortega Reyna ${ }^{34}$
}

${ }^{1}$ Escuela de Psicología, Universidad César Vallejo. Perú

${ }^{2}$ Unidad de Posgrado, Universidad Nacional Mayor de San Marcos. Perú

${ }^{3}$ Universidad Nacional Federico Villareal. Perú

${ }^{4}$ Universidad San Ignacio de Loyola. Perú

\begin{abstract}
Resumen: La presente investigación tiene como objetivo describir los niveles de depresión en una población de pacientes con insuficiencia renal crónica en tratamiento de hemodiálisis, con el fin de evaluar si existen diferencias significativas de acuerdo a factores sociodemográficos y tiempo de hemodiálisis. El estudio es descriptivo-comparativo, el instrumento aplicado fue el inventario de depresión de Beck. La muestra estuvo formada por 503 pacientes de la ciudad de Lima y del interior del país Los resultados muestran que el nivel de depresión predominante es el leve (48.9\%), que a mayor rango de edad y menor grado de instrucción la tendencia a presentar depresión es mayor; de acuerdo al sexo los pacientes con mayor tendencia a la depresión son los varones e igualmente los viudos y divorciados; de acuerdo al tiempo de diálisis la tendencia a aumentar la depresión es conforme aumenta el tiempo de diálisis. Se concluye que los niveles de depresión tienden a presentarse de forma significativa de acuerdo a los factores sociodemográficos $(p<0.00)$.
\end{abstract}

Palabras clave: depresión, pacientes crónicos renales, factores sociodemográficos, hemodiálisis, trastornos del estado de ánimo

\begin{abstract}
The objective of this research is to describe the levels of depression in a population of patients with chronic renal failure on hemodialysis treatment, in order to assess whether there are significant differences according to sociodemographic factors and time of hemodialysis. The study is descriptive-comparative, the instrument applied was Beck's depression inventory. The sample consisted of 503 patients from the city of Lima and from the interior of the country. The results show that the predominant level of depression is mild (48.9\%), that the greater the age range and the lower the level of education the tendency to present depression is greater; according to sex, the patients with the greatest tendency to depression are men and also widows and divorcees; According to the dialysis time the tendency to increase the depression is as the dialysis time increases. It is concluded that the levels of depression tend to present in a significant way according to sociodemographic factors $(p<0.00)$.
\end{abstract}

Keywords: depression, chronic renal patients, sociodemographic factors, hemodialysis, mood disorders

Cómo citar este artículo:

Vallejos Saldarriaga, J.F., \& Ortega Reyna, E.Z. (2018). Niveles de depresión y factores sociodemográficos en pacientes con insuficiencia renal crónica en tratamiento de hemodiálisis en Perú. Ciencias Psicológicas, 12(2), 205-214. doi: https://doi.org/10.22235/cp.v12i2.1683.

Correspondencia: José Francisco Vallejos Saldarriaga, Universidad César Vallejo - Perú; e-mail: jvallejoss@ucv.edu.pe. Eris Zoila Ortega Reyna, Universidad Nacional Federico Villareal - Perú; e-mail: zoilaortega@hotmail.com 


\section{Introducción}

La enfermedad renal crónica (ERC) es considerada un problema de salud pública en el mundo (Herrera, Palacios, \& Hernández, 2014), por lo que los gobiernos y entidades de salud mantienen dentro de sus lineamientos de salud, una especial mirada para esta enfermedad; promoviendo programas de atención y tratamiento entre los que se encuentran las de remplazo renal para los diagnósticos de Insuficiencia Renal Crónica IRC, no siendo los más equitativos económica y socialmente en las mayoría de países llamados en proceso de desarrollo, entre los que se encuentra el Perú (Hurtado \& Aréstegui 2007).

Perales-Montilla, Duschek y Reyes-del Paso (2013) mencionan que el estrés, la ansiedad y la depresión se asocian a una mayor morbilidad y mortalidad por diversas enfermedades, muchas de las cuales pueden presentar afectividad negativa, malestar emocional y predisposición a experimentar disgusto, ansiedad, tristeza, hostilidad-ira, culpabilidad, miedo, insatisfacción con uno mismo, mayor autocrítica, valoración negativa de uno mismo, del mundo y el futuro, etc. Esta sintomatología predice una mayor frecuencia de quejas somáticas, desarrollo de trastornos mentales y otras dolencias físicas, siendo la ansiedad y depresión factores que pueden intensificar y empeorar el curso y la evolución de la enfermedad e interferir con su tratamiento y la percepción de los síntomas.

Los pacientes que sufren la pérdida irreversible de la función renal denominada clínicamente, insuficiencia renal crónica (IRC) o enfermedad renal crónica (ERC), presentan alteraciones significativas a nivel físico, biológico, psicológico, familiar y social. Alteraciones entre las cuales se destaca la sintomatología depresiva y ansiosa como reacción ante la crisis y afrontamiento frente a la enfermedad y procesos de tratamiento (Alarcón, 2004; Cerna, Bocanegra, Jiménez, \& Díaz, 2014; Medina \& Espinach, 2013).

La insuficiencia renal crónica aparece como principal forma de reacción frente a los problemas que trae la enfermedad renal y sus tratamientos (Ortega \& Pérez, 2004); obteniendo puntuaciones significativamente mayores que la población en general, como lo refieren también Martínez, Martínez y Andrés (2007), una enfermedad que afecta no solo la vida cotidiana sino la calidad de vida del que la padece (Carré, 2006; Douthat, 2007), evidencia una alta prevalencia en su contexto según lo mani- fiestan Pérez-Domínguez, et al. (2102).

La depresión aparece en las distintas etapas de progresión de la enfermedad renal, con alta prevalencia sobre todo en pacientes renales crónicos en tratamientos de hemodiálisis; su sintomatología psicosomática y emocional, afecta el proceso y manejo de la enfermedad renal y tratamiento, las consecuencias emocionales e interacciónales en la vida del paciente repercuten significativamente en su adherencia y calidad de vida (Pérez-Domínguez, et al., 2012).

La depresión es catalogada como un trastorno afectivo que se asocia con un sentimiento de pérdida. Según Rubio, Sánchez, Jiménez, Kanahan y Oria (2012), es una característica asociada a diversos procesos y eventos que experimentan los pacientes con diagnósticos de insuficiencia renal, especialmente de IRC, alteración que afecta su autonomía, cambios de hábitos, relaciones interpersonales, de pareja, rol familiar, contexto laboral y social; esta coyuntura de salud demanda la atención clínica médica en resguardo y salvaguarda de la vida; la intervención en salud mental y psicológica contribuyen para que el paciente conduzca adecuadamente su enfermedad (García \& Calvanese, 2008).

Los tratamientos que se brindan a un paciente que padece de IRC, son sustitutivos parciales de la función renal, incluyen situaciones de dependencia, manifestaciones psicosomáticas, cuidados alimentarios, ingesta limitada de líquido, estricto régimen terapéutico de ingesta medicamentosa, cuidados físicos, limitaciones en sus actividades, miedos entre otras manifestaciones. Situaciones que no favorecen la asimilación de una adecuada conciencia de enfermedad y conlleva la aparición de reacciones ansiosas y depresivas que afectan la evolución de su estado de salud y por ende de su calidad de vida, como lo mencionan Orellana y Munguía (2008); Pérez-Domínguez, et al. (2102); Perales-Montilla et al. (2013).

En hemodiálisis, el $71 \%$ de pacientes atendidos con IRC, son de la región de Lima y sólo el 29\% de provincias (Arquinigo, 2008). En los últimos 15 años EsSalud, entidad paraestatal del sistema de salud peruano, ha atendido al porcentaje mayoritario de la población renal que incrementó la terapia de reemplazo renal-hemodiálisis. Bajo esta situación se ha podido apreciar que en las instituciones públicas Ministerio de Salud (MINSA) y Seguro Social (EsSalud) así como en el sector privado, el incremento no solo afecta y demanda el apoyo del ámbito clínico y/o médico, sino que demanda un 
abordaje multidisciplinario, donde el apoyo psicológico y de salud mental integral es importante por cuanto promueve cambios significativos en la vida de los pacientes y sus familias. En este contexto, emergen cuadros clínicos como la depresión y la ansiedad por el presente y futuro (Carré, 2006; Douthat, 2007), en algunos casos como reacción o episodio, en otros como un trastorno afectando no sólo su vida cotidiana sino su calidad de vida, lo cual es relevante estudiar en estas poblaciones para poder atenderlas de la mejor manera y humana posible.

La enfermedad renal crónica, es una enfermedad que exige una mayor atención de la familia $\mathrm{y}$ del personal de salud en todas las etapas de su

Tabla 1

Depresión y hemodiálisis desarrollo. Los pacientes se adaptan a sus tratamientos de reemplazo o sustitución de la función renal, lo que implica estar sometido a cambios clínicos, alteraciones psicológicas y mentales. Es necesario tener en cuenta que los diagnósticos clínicos de pacientes renales, en un porcentaje mayoritario, tienen relación con la prevalencia de Diabetes Mellitus, Hipertensión Arterial, lupus, vasculitis y otros diagnósticos clínicos médicos asociados, los mismos que también traen consigo sintomatología clínica y emocional que incluyen a la depresión en porcentajes significativos, a los que se agregan los efectos propios de la IRC y tratamientos. Los avances de la investigación sobre el tema se presentan en tabla 1.

Autores y año
Álvarez, Fernández, Vázquez, Mon,
Sánchez, \& Rebollo (2001)
Ortega y Pérez (2004)
Martínez, Martínez y Andrés (2007)
Carré, (2007)
Aguilera, P. (2007)
García, Fajardo, Guevara, Gonzales
y Hurtado (2008)
García y Calvanese (2008)
Páez, Jofré, Marcos, Azpiroz, y De
Bortoli (2008)

Amador, Pons, Espinosa (s/f)

Esquivel, Prieto, López, Ortega, Martínez, y Velazco (2009)

Perales-Montilla, García-León y Reyes del Paso, (2012) Pérez-Domínguez, RodríguezPérez, García-Bello, Buset-Ríos, Rodríguez-Esparragón, ParodisLópez, Rodríguez- Pérez (2102) Cerna, Bocanegra, Jiménez y Díaz (2014)

Gómez, L., Gracia, N., Manresa, M., Existe alta prevalencia de ansiedad y depresión en pacientes con enfermedad renal crónica en Lozano, S., \& Chevarría, J. (2015)

Villanueva y Casas (2015)

Nieto, M. (2017)

Rojas, Y., Ruiz, A., y González, R. (2017)

Aportes

La ansiedad y la depresión están asociadas a la sintomatología orgánica y a repercusiones en el tratamiento de hemodiálisis.

La depresión aparece como principal forma de reacción frente a los problemas que trae la enfermedad y sus tratamientos, todos los pacientes de la muestra con o sin instrucción padecen de sintomatología depresiva.

Obtienen puntuaciones significativamente mayores que la población teórica, en depresión, ansiedad y estrés.

La depresión aparece como una reacción, llegando en muchos casos a ser un trastorno, afectando no solo su vida cotidiana sino su calidad de vida.

En un estudio en hospital Chileno en 93 pacientes se encontró que el 63\% de la población presenta algún grado de depresión; el $15.7 \%$ posee depresión de tipo leve, el $42.8 \%$, moderada y el $5.2 \%$ grave. La mayor prevalencia se encontró en hombres entre 15 a 30 años y entre 47 a 62 años que llevan tres años en tratamiento, procedentes de zonas urbanas, sin pareja estable, que perciben bajos ingresos económicos mensuales, con baja escolaridad, con familias monoparentales e intentaron alguna conducta suicida después de conocer el tratamiento a seguir.

Los síntomas depresivos y su severidad están asociados a una mayor ganancia de peso interdialítica y a un mayor nivel de potasio sérico pre diálisis en pacientes con terapia crónica de hemodiálisis (conciencia de enfermedad).

La dependencia a las "máquinas" y al personal de salud, las restricciones en la dieta, los cambios en las relaciones sociales y familiares y la situación laboral, entre otras", generan trastornos emocionales y mayores niveles de depresión y ansiedad que se asocian a una calidad de vida deficiente. La ansiedad y depresión es mayor en los enfermos renales crónicos de más edad y durante los primeros meses de hemodiálisis, agregando que los pacientes en tratamiento de Hemodiálisis (HD) presentan frecuentemente 'trastornos emocionales adaptativos mixtos conformados por la presencia conjunta de ansiedad y depresión. El grado de depresión es mayor en los pacientes sin actividad laboral.

Se obtuvo que el 54,29\% de los pacientes tienen depresión en algún grado (34.29\% leve, 14.29\% moderada-severa, $5.71 \%$ severa).

La calidad de vida del paciente con insuficiencia renal crónica terminal es menor a $50 \%$ en las áreas físico y mental, el $53.7 \%$ de los pacientes tienen niveles más elevados de depresión ( $18,2 \%$ Leve, $20 \%$ Moderada y $14.5 \%$ Severa).

La depresión es el principal predictor de la calidad de vida relacionada con la salud - CVRS y se encuentra asociada negativamente con todas sus dimensiones.

La depresión es un trastorno afectivo que se asocia con un sentimiento de pérdida", manifestaciones que presentan los pacientes con problema renal al constatar que pierden autonomía, su rol familiar y desempeño laboral. Aparece con alta prevalencia en pacientes con enfermedades renales crónicas. Encontraron el $57,78 \%$ de pacientes con depresión y $65,19 \%$, con ansiedad, la frecuencia de depresión y ansiedad en los pacientes diabéticos tipo 2 fue elevada

hemodiálisis. Las mujeres de mayor edad evidencian más depresión y la ansiedad.

En 39 pacientes en tratamiento de hemodiálisis se encontró que a mayor depresión en los pacientes se relacionaba con un menor nivel de calidad de vida.

El nivel de depresión fue medio en las áreas cognitiva reflejado en la falta de concentración y deterioro de las relaciones interpersonales, así como en el área física con problemas de sueño, pérdida de peso y dolores corporales difusos.

En una muestra de 36 casos encontraron síntomas depresivos (48\%). La depresión se relacionó con menor adherencia específicamente en el control de ingesta de alimentos/líquidos y seguimiento médico. 
Teniendo en cuenta el reporte que la literatura hace sobre el impacto y efectos de la depresión en los pacientes crónicos renales, donde se evidencia el incremento no sólo de los síntomas sino también del estado general del paciente, tales como la alteración de las funciones cognitivas, mentales, emocionales y calidad de vida, se considera importante explorar los niveles de depresión en poblaciones de pacientes con IRC con tratamientos de hemodiálisis, a fin de conocer sus diferencias de acuerdo a condiciones sociodemográficas, lo que permitirá tomar decisiones terapéuticas y medidas interdisciplinarias de prevención y promoción de la salud de estos pacientes.

\section{Metodología}

En la presente investigación se ha trabajado con un diseño no experimental de tipo transversal.

\section{Participantes}

La muestra está conformada por 503 pacientes con el diagnóstico de insuficiencia renal crónica, en tratamiento de diálisis intermitentes de 10 centros de hemodiálisis de la ciudad de Lima, hombres y mujeres con un rango de edad entre los 20 a 92 años, con una M: de 52 años y una DE: de 15 y con más de un mes de permanencia en los centros hospitalarios del estudio.

Se excluyeron los pacientes con diagnóstico clínico de complicaciones severas, lo que tenían síntomas físicos y clínicos que impedían la realización de la evaluación, aquello con diagnóstico de VIY y los que evidenciaran síntomas clínicos de enfermedad mental -que no fuera trastorno del estado de ánimo-.

La muestra se caracteriza por tener un ligero predominio de hombres en relación a las mujeres (H: 56.7\%; M: 43.3\%), con preeminencia de personas casadas $(54.9 \%)$, seguido de los solteros $(27.4 \%)$, los menores porcentajes se presentan entre los divorciados $(2.8 \%)$ y separados $(2.2 \%)$. En el grado educativo se observa un predominio de la educación secundaria (33.2\%), seguido de primaria incompleta $(17.5 \%)$ y superior incompleta $(11.3 \%)$. En relación al lugar o región de procedencia, el mayor porcentaje es de la ciudad de Lima (43.7\%), seguido de Junín $(6.6 \%)$ y Ayacucho (6\%). La menor prevalencia de pacientes que se han estudiado son de las regiones de: Tacna ( $0.6 \%)$, Huancavelica $(0.8 \%)$ y Ucayali $(0.8 \%)$. En relación al tiempo de diálisis, el mayor porcentaje de pacientes llevan entre 2 y 3 años $(19.5 \%)$.

La población del estudio fue seleccionada a través de un muestreo no probabilístico de tipo intencional, la elección de los casos se realizó en función de la disposición a participar en la investigación y con posibilidad de rechazar su participación o discontinuarla (Hernández, Fernández, \& Baptista, 2014).

La evaluación de los pacientes se realizó con conocimiento de los mismos, dando aprobación para la toma de la prueba y se les informó en detalle acerca de los motivos y objetivos de la presente investigación; así mismo se les señaló que la identidad de los participantes era estrictamente confidencial.

\section{Instrumento}

La prueba utilizada para esta investigación es el inventario de depresión de Beck o BDI (Beck, Epstein, Brown, \& Steer, 1988) que está compuesto por 21 ítems, miden niveles moderados, medios, altos y severos signos de depresión, tiene validez de contenido, se puede evaluar sobre todo los síntomas depresivos cognitivos de los cuales 10 de sus 21 ítems evalúan síntomas cognitivos, mientras que sus ítems evalúan síntomas fisiológicos, 3 síntomas anímicos y 3 síntomas motivacionales.

Tras una versión revisada realizada en 1979, se pudieron sistematizar 4 alternativas de respuestas para cada ítem, cada una con orden desde menor a mayor gravedad, su contenido destaca por el componente cognitivo, ya que los síntomas de la depresión representan un $50 \%$ de lo que es la puntuación total del cuestionario, y en donde se observan síntomas de tipo somático, como segundo signo se observa el de tipo vegetativo, en distribución refiere que 15 ítems hacen referencia a síntomas psicológicos cognitivos, mientras que 6 síntomas de tipo somático - vegetativo.

Beck (1976) desarrolló esta prueba basada en su teoría cognitiva y clasificó en áreas y subdimensiones, estructurándose según se detalla en la tabla 2.

Cada ítem se valora de 0 a 3 puntos en función de la alternativa escogida, luego de sumar se obtiene una puntuación total cuyo rango fluctúa de 0 a 63 y que cuantifica la presencia y gravedad de sintomatología depresiva mas no un diagnóstico, 
Tabla 2

Áreas y sub dimensiones del BDI

\begin{tabular}{lll}
\hline Áreas & \multicolumn{1}{c}{ Evalúa } & \multicolumn{1}{c}{ Sub dimensiones } \\
\hline \multirow{2}{*}{ Afectiva } & \multirow{2}{*}{ Estado de animo } & 1. Tristeza \\
& & 5. Sentimientos de culpa \\
& & 10.Predisposicion para el llanto \\
& & 11. Irritabilidad \\
Motivacional & Satisfacción personal & 4. Satisfacción \\
& & 9. Ideas suicidas \\
& & 2. Pesimismo frente al futuro \\
& & 3. Sensación de fracaso \\
Cognitiva & Estado de percepción de la & 6. Expectativa de castigo \\
& persona & 7. Auto disgusto \\
& & 13.Indecisión \\
Conductual & & 14.Autoimagen deformada \\
& & 20.Preocupaciones somáticas \\
& Alteración de la conducta & 8. Autoacusaciones \\
& & 12 Alejamiento social \\
Física & & 15 lentitud para trabajar \\
& & 17 Fatigabilidad \\
& Estado físico y fisiológico de & 16 Pérdida de peso \\
& la persona & 19 Pérdida de apetito \\
& & 21 Perdida de peso deseo sexual \\
\hline
\end{tabular}

los puntajes o cortes aceptado para la graduar la intensidad o presencia de la depresión es como sigue: 0-10: Sin depresión; 11-20: depresión leve; 21-30: depresión moderada; más de 31 : depresión grave.

La prueba ha demostrado valores de confiabilidad que oscilan entre los 0.76 y 0.95 en muestras de pacientes psicopatológicos, entre 0,73 y 0,93 en muestras de la población general, y entre 0,78 y 0,92 en muestras de estudiantes universitarios (Beck et al., 1988).

En validez la prueba ha demostrado aceptables índices de validez, en la medida que sus ítems correlacionan de forma moderada o alta con otras medidas de depresión en distintos tipos de población (pacientes psicopatológicos, adultos de la población general, adolescentes, estudiantes universitarios, ancianos, pacientes médicos, etc.).

Adicionalmente y mediante una ficha sociodemográfica se obtuvieron datos de la historia clínica, tales como: Edad, sexo, lugar de nacimiento, estado civil, grado de instrucción, tiempo de diálisis, fecha de evaluación, respetando el estado de anonimato.

\section{Procedimiento}

Se procedió a la coordinación de los permisos con las entidades de salud seleccionadas para la investigación. Se propuso las fechas para la selección de la muestra, coordinando con los médicos jefes de los servicios y responsables de las
Unidades de Diálisis mencionadas, para el acceso a las historias clínicas psicológicas. Se procedió a la obtención del Consentimiento Informado de los pacientes seleccionados.

El BDI fue aplicado por los psicólogos y colaboradores de 10 Servicios de Hemodiálisis de manera individual y algunas veces en pequeños grupos de 2 o 3 pacientes, dependiendo de su estado físico y emocional

Los derechos, intimidad, bienestar y dignidad de los pacientes han sido protegidos de acuerdo a los principios establecidos por la APA (2010) y el Código de Ética del Colegio de Psicólogos del Perú ( $\mathrm{s} / \mathrm{f}$ ). Asimismo, la investigación ha sido aprobada por el Comité de Ética de la Escuela de Psicología y Dirección de Investigación de la Universidad César Vallejo.

\section{Resultados}

En relación a los niveles de depresión, el $48.9 \%$ de los participantes mostró un nivel de depresión leve, el $40.2 \%$ un nivel moderado y $3.2 \%$ presenta un nivel severo de depresión. Los niveles de depresión de acuerdo al sexo se distribuyen: Mujeres: leve $41.7 \%$, moderado 47.7 y severo 3.7; en tanto, en Varones el nivel leve se sitúa en el $54.4 \%$, moderado $34.4 \%$ y severo en el 2.8\% (ver figura 1). 


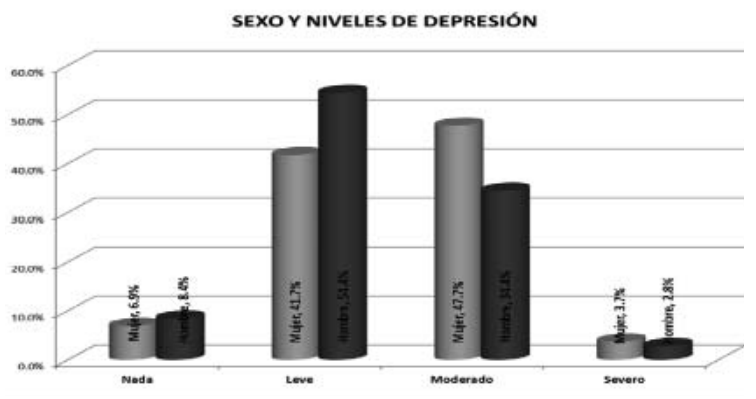

Figura 1

Porcentaje de pacientes en cada uno de los niveles de depresión de acuerdo al sexo
Tomando en cuenta el grado de instrucción, los pacientes renales con menor nivel educativo presentan una mayor tendencia a la depresión que los de otros niveles educativos (ver figura 2).

En relación al estado civil, se da un mayor porcentaje de pacientes viudos con depresión moderada, siguiendo divorciados y casados (ver figura 3 ).

En cuanto a la edad hay un incremento de la incidencia de la depresión a nivel moderado en la medida que se envejece; en tanto, los niveles de depresión leve son mayores a menor edad y luego

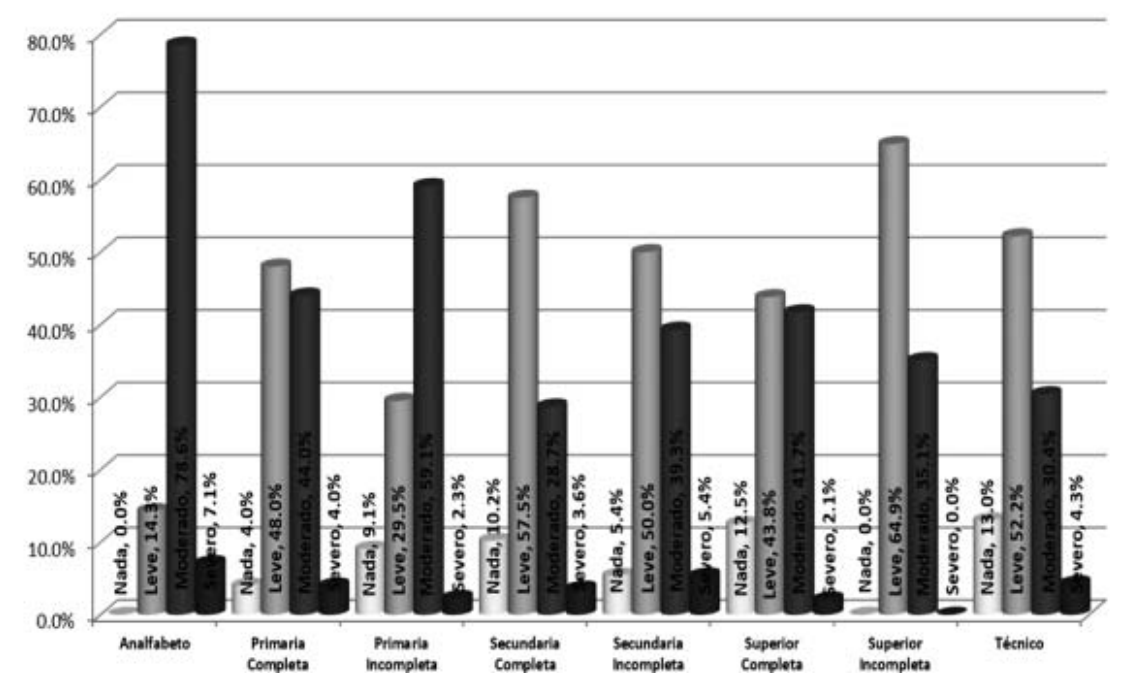

Figura 2

Porcentaje de pacientes en cada uno de los niveles de depresión de acuerdo al grado de instrucción

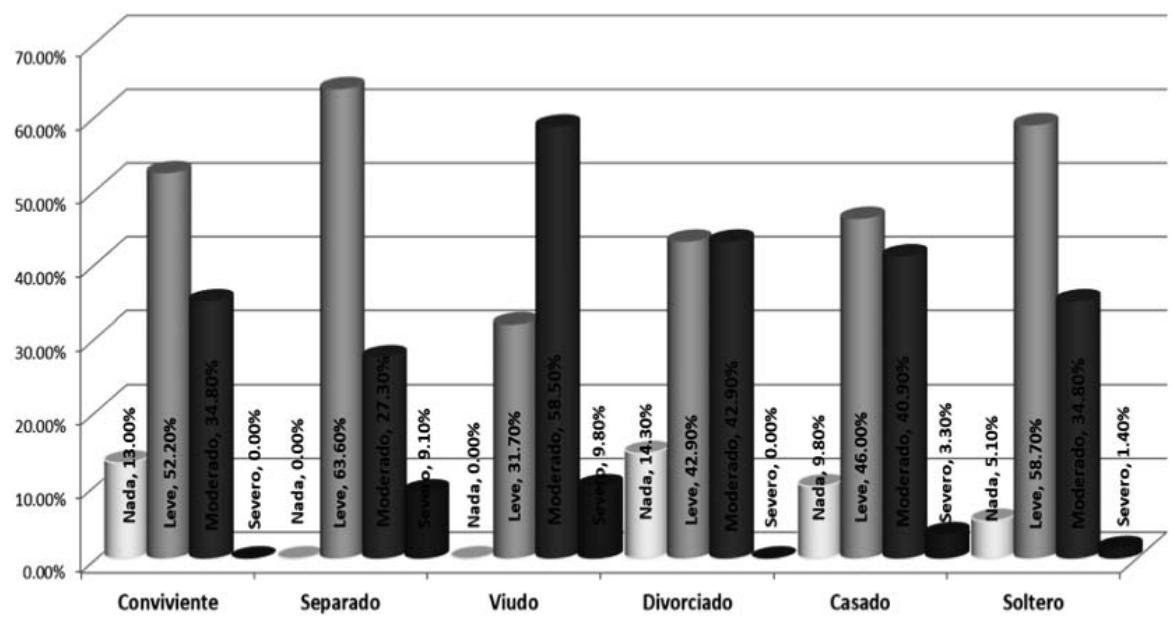

Figura 3

Porcentaje de pacientes en cada uno de los niveles de depresión de acuerdo al estado civil 
se incrementan exponencialmente a partir de los 90 años, para esta población (ver figura 4).

De acuerdo a la procedencia, las regiones que presentan los niveles más altos de depresión son Amazonas, Huancavelica, Ica Callao, Apurímac, la Libertad, San Martin, Loreto y Ayacucho. En la figura 5 se aprecia que varias regiones tienen niveles de más del $40 \%$ de depresión leve y algunas también moderada.

Los niveles de depresión de acuerdo al tiempo de diálisis presentarían una elevación en el nivel moderado, hay variaciones sobre las que habría que profundizar especialmente en el nivel leve, que se eleva considerablemente entre los 12 y los 15 años y luego de los 20 años de tratamiento de diálisis (ver figura 6).
Los niveles de depresión se diferencian de forma significativa teniendo en cuenta los diferentes rangos y el tiempo de diálisis $(p=0.00, \mathrm{X}: 2.3$ años), salvo en el rango de 16 a 19 años de diálisis.

Los niveles de depresión se diferencian de forma significativa teniendo en cuenta el sexo ( $p: 0.00)$; el estado civil ( $p: 0.00)$ y el nivel de instrucción de los pacientes estudiados ( $p: 0.00)$.

\section{Discusión}

La Enfermedad Renal Crónica (ERC) es considerada un problema de salud pública en el mundo (Herrera et al., 2014). En el Perú e internacionalmente se le ha estudiado en relación con otras variables. Uno de los aspectos trabajados tanto en

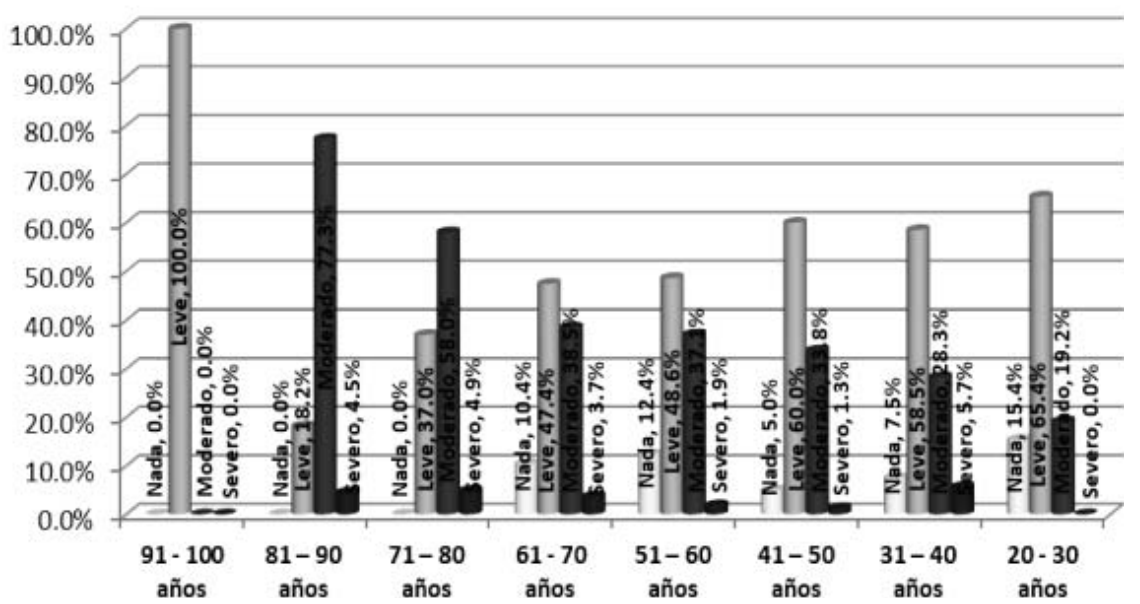

Figura 4

Porcentaje de pacientes en cada uno de los niveles de depresión de acuerdo a la edad

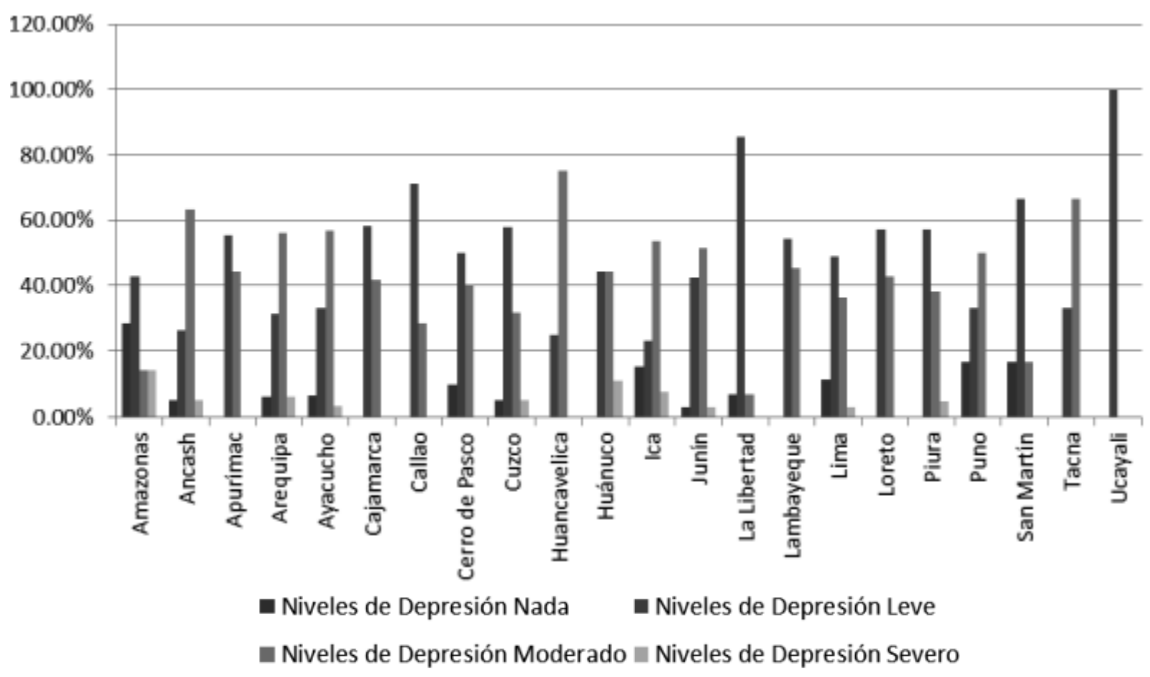

Figura 5

Porcentaje de pacientes en cada uno de los niveles de depresión de acuerdo a la procedencia de los pacientes 


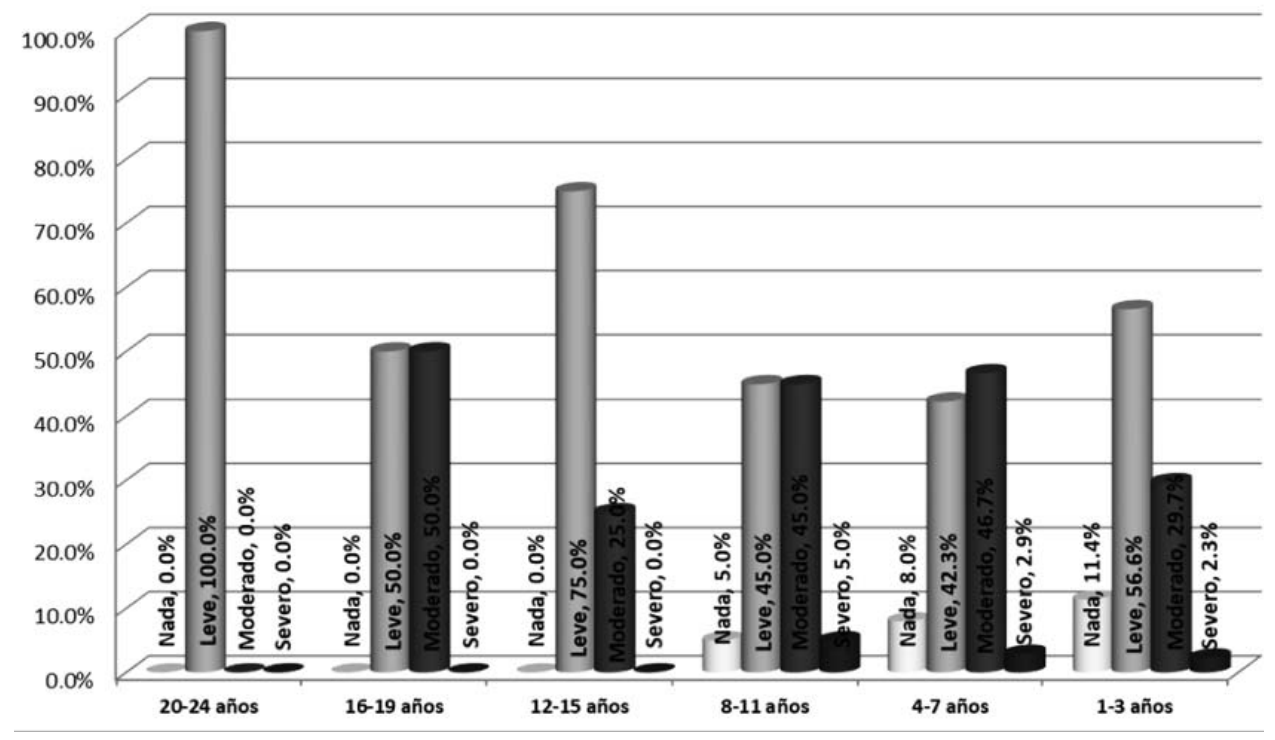

Figura 6

Porcentaje de pacientes en cada uno de los niveles de depresión de acuerdo al tiempo de diálisis

los contextos clínicos médicos como psicológicos es la depresión en la población de pacientes renales crónicos, quienes la cursan en diferentes niveles de intensidad, dependiendo de determinadas variables que se presentan en muchos casos con el papel de moderadoras. La presente investigación muestra datos en relación a la depresión en los pacientes con enfermedad renal crónica en tratamiento de hemodiálisis y las variables sociodemográficas estudiadas.

La población de pacientes renales y tipos de tratamientos requiere atención especializada generalmente brindada en hospitales nivel III y IV del Seguro Social-EsSalud y Hospitales nivel II y III del Ministerio de Salud-MINSA., incluidos centros subcontratados de ambas instituciones, especialmente en Lima. Se puede observar que se tienen muestras que van desde los 20 a los 92 años coincidiendo con lo que refiere Álvarez et al. (2001) y Perales-Montilla, García-León y Reyes del Paso, (2012) que la depresión se encuentra presente en todas las etapas, procesos y repercusiones del tratamiento de hemodiálisis y muchas veces está asociada a sintomatología orgánica, afectando la calidad de vida de estos pacientes. Cabe destacar que un $7.8 \%$ no presenta depresión, hecho que podría estar relacionado con diferentes recursos de la personalidad y el apoyo familiar y social.

Por otro lado, los pacientes varones muestran porcentajes y niveles próximos a los obtenidos en la población femenina especialmente en los que hace a los niveles Leve y Moderado. Focalizando en la población masculina, ésta tiene un porcentaje mayor en el nivel de depresión Leve, en tanto las mujeres en el Moderado, lo que concuerda con los resultados encontrados por Gómez et al. (2015) quienes además refieren que son las mujeres de mayor edad que evidencian mayor depresión. El alto nivel de depresión leve en los varones se debería a un bagaje de respuestas que tienen que ver con un contexto donde las condiciones neuropsicológicas, la cultura y el ambiente psicosocial, entre otros, no auspiciarían la expresión emocional directa como llorar ("los hombres no lloran" refrán popular), sin embargo, éstas influirían en aspectos como el cambio del rol en la familia, en el desempeño laboral, en la autosuficiencia económica y especialmente en su autoimagen (Pérez-Domínguez, et al., 2102) pudiendo encontrarse en la génesis de su aparición.

El resultado en mujeres podría estar relacionado con una mayor predisposición y ventaja social a evidenciar y mostrar externamente su dolor emocional y físico. Es importante destacar que en ambos sexos la depresión es fluctuante con rangos superiores al $30 \%$, en donde, de cada 100 casos, 40 se verían afectados a nivel leve y moderado y sólo 3 de cada 100 se verían afectados por la depresión de una manera severa, evidenciándose un rango de aproximadamente $6 \%$ que no presentan depresión.

Los niveles de depresión de acuerdo al estado civil, muestran una mayor tendencia a la depresión moderada en los pacientes viudos, siguiendo los divorciados y solteros; estos hallazgos se pueden entender en el sentido que los pacientes, en especial los adultos mayores, estarían más afectados 
en muchos casos por la ausencia de la pareja y/o acompañante significativo, como fuente de apoyo para manejar la enfermedad y concurrir a sus tratamientos, siendo la depresión un trastorno afectivo asociado con pérdidas y ansiedad (PérezDomínguez et. al, 2002; Rubio et al., 2012). Estos resultados estarían en relación a los hallazgos que evidencian que los niveles de depresión tienen relación con la edad y también con la forma como clínicamente evolucione la enfermedad -los pacientes de la tercera edad presentan una tendencia a aumentar los índices depresivos conforme a la edad, especialmente entre los 71 a 100 años- y en particular entre 71 a 90 años, rango en los cuales la depresión tiende a incidir mucho más.

En cuanto a los niveles de depresión de acuerdo a la región de procedencia -según lugar de nacimiento-, se observó que, las regiones que presentan los niveles más altos y graves de depresión son: Amazonas, Huancavelica, Ica Callao, Apurímac, la Libertad, San Martin, Loreto y Ayacucho; estos resultados podrían estar relacionados con los hallazgos de los estudios del Instituto Nacional de Salud Mental que detecta zonas de mayor incidencia de la depresión en el Perú. Según estos estudios los niveles más altos de depresión se dan en zonas de sierra y selva y otras ciudades del interior del país, en niveles moderados y severos, esto podría relacionarse con la migración y los limitados recursos en esas zonas para tratamiento y las transferencias a la seguridad social, a lo que se suman otras comorbilidades y problemas de apoyo social y económico.

En relación al tiempo de tratamiento de diálisis, se distingue una cierta tendencia a la aumentar con el tiempo; sin embargo, hay variaciones que podrían obedecer a otros factores médicos, familiares y sociales, como en el rango de 20-24 años de tratamiento de la enfermedad que podría estar influyendo en el deterioro del organismo (Álvarez, Fernández, Vázquez, Mon, Sánchez, \& Rebollo, 2001). Estas diferencias significativas podrían estar relacionadas con la respuesta al proceso del diagnóstico, procedimientos médicos, comorbilidad y limitación funcional, perdida de la independencia y el tipo de tratamiento de la enfermedad renal, incluyendo el impacto emocional, duelo psicológico por la pérdida de la salud y cambio en los estilos de vida personal y familiar (García \& Calvanese, 2008).

Los niveles de depresión de acuerdo al grado de instrucción son coincidentes con lo que citan Ortega y Pérez (2004), en cuanto a que los pacientes renales de menor nivel educativo presentan una mayor tendencia a la depresión que otros niveles educativos. Frente a estos resultados se podría plantear que, el estatus de desarrollo, desempeño cotidiano y laboral les haría más vulnerables frente a la enfermedad y el tratamiento de hemodiálisis, por cuanto involucra elementos estresores relacionados con su autonomía y el rendimiento, dependencia de la máquina, tiempo de tratamiento, horas y lugar de tratamiento, restricciones en la dieta, relaciones sociales, familiares y situación laboral, entre otras, generando trastornos emocionales y mayores niveles de depresión y ansiedad que se asocian a una calidad de vida deficiente (García \& Calvanese, 2008).

Los niveles de depresión en edades más altas -tal el caso de pacientes mayores de 90 años- alcanzan los porcentajes más altos en el nivel leve, lo que puede estar relacionados con la comorbilidad, cuidado y trato del paciente y red familiar-social. La ansiedad y depresión es mayor en los enfermos renales crónicos de más edad y durante los primeros meses de hemodiálisis, agregando que la presencia de la depresión es también significativa en los pacientes más jóvenes, aunque los pacientes renales cursan síntomas depresivos sin importar la edad y modalidad de su tratamiento (Páez et al., 2008; Ortega \& Pérez, 2004).

La presente investigación presenta limitaciones en el tipo de muestreo realizado, no aleatorio, lo que no le permite tener validez externa; sin embargo, el tamaño muestral amplio puede permitir un menor error y una mejor aproximación. Es importante realizar estudios con una mayor diversidad de variables sociodemográficas que permita precisar el papel de ellas y sobre el cuadro clínico de depresión y enfermedad renal, profundizando aspectos que pueden estar incidiendo en el comportamiento de las variables estudiadas.

\section{Conclusiones}

- La depresión es parte del contexto clínico emocional del paciente renal crónico, observándose desde el diagnóstico, en las etapas de la enfermedad y en el tratamiento de diálisis.

- La población de varones con la enfermedad renal crónica en diálisis presenta una mayor tendencia a una depresión leve, las mujeres tienden hacia depresiones moderadas.

- Mientras mayor es el rango de edad y menor grado de instrucción la tendencia a mantener depresión en el paciente renal crónico es mayor.

- Los niveles de depresión de acuerdo al tiempo 
de diálisis son moderados, pero tienden a aumentar conforme aumenta el tiempo de diálisis.

- Existen diferencias de forma significativa en los niveles de depresión de acuerdo a los factores sociodemográficos y clínicos.

\section{Referencias}

Alarcón, A. (2004). La depresión en el paciente renal. Rev. Colomb. Psiquiatr.;33 (3). Recuperado de: http:// www.scielo.org.co/scielo. php?script=sci_arttext\&pid $=$ S0034-74502004000300005

Álvarez, F., Fernández, M., Vázquez, A., Mon, C., Sánchez, R., \& Rebollo, P. (2001). Síntomas físicos y trastornos emocionales en pacientes en programa de hemodiálisis periódicas. Revista de Nefrología; 21(2), 191-199.

APA (2010). American Psychological Association ethical principles of psychologist and code of conduct. Recuperado de http://www.apa.org/ethics/code/index.aspx

Arquinigo, G. (2008). Capacidad de autocuidado del paciente en hemodiálisis periódica del Centro de Hemodiálisis de EsSalud, 2006- 2007. [Tesis inédita para optar el título de Magister en Enfermería]. Universidad Nacional Mayor de San Marcos.

Beck, A. (1976). Cognitive Therapy and the emotional disorders. New York: International University Press.

Beck, A., Epstein, N., Brown, G., \& Steer, R. (1988). An inventory for measuring clinical anxiety: psychometric properties. Journal of Consulting and Clinical Psychology, 56, 893-897.

Carré, V. (2006). Relación entre el nivel de Inteligencia emocional y el nivel de depresión en los pacientes de la Unidad de Hemodiálisis del Hospital Víctor Lazarte Echegaray. [Tesis inédita para optar el Grado de Licenciada en Psicología]. Universidad César Vallejo. Trujillo.

Cerna, A., Bocanegra, M., Jiménez, F., \& Díaz, C. (2014). Frecuencia de depresión y ansiedad en pacientes con diabetes tipo 2 atendidos en un hospital general de Chiclayo. Revista Herediana; 25: 196-203.

Douthat, W. (2007). La enfermedad renal crónica y su impacto en la salud pública. Experimental Médicine. 2007; 25(2/4), 5960. Recuperado de http://bases.bireme.br/cgi-bin/wxislind. exe/iah/online/

García, H. \& Calvanese, N. (2008). Calidad de vida percibida, depresión y ansiedad en pacientes con Tratamiento sustitutivo de la función renal. Revista Psicología y salud, 18 (1); 5-15. Recuperado de http://www.redalyc.org/ pdf/291/29118101.pdf

Gómez, L., Gracia, N., Manresa, M., Lozano, S., \& Chevarria, J. (2015). Prevalencia de ansiedad y depresión en pacientes de hemodiálisis. (Spanish). Enfermería Nefrológica; 18(2), 112-117. Recuperado de http://eds.b.ebscohost.com/ ehost/pdfviewer/pdfviewer?vid=6\&sid=35436e3c-73c94c17-b9d7-17df5c72b22e\%40sessionmgr120\&hid=122

Herrera, P., Palacios, M., \& Hernández, A. (2014). Alta tasa de interrupción de hemodiálisis en pacientes del Hospital Nacional 2 de Mayo de Perú. Revista de Nefrología, diálisis y trasplante. 34(2), 94-98. Recuperado de http://www. scielo.org.ar/scielo.php?pid=S234685482014000200008 \&script $=$ sci_arttext

Hernández, R. Fernández, C., \& Baptista, P. (2014). Metodología de la investigación. $6^{\text {a }}$ edición. Mc Graw Hill: México.
Hurtado, A. \& Aréstegui, E. (2007). Enfermedad Renal Crónica Terminal y Factores de Riesgo en Perú. Análisis Costo Beneficio de la Prevención. Servicio de Nefrología Carlos Monge Cassinelli. Hospital Nacional Arzobispo Loayza, Universidad Peruana Cayetano Heredia. Lima Perú.

Medina, A. \& Espinach, M. (2013). Depresión y ansiedad en usuarios mayores de 18 años del Servicio de Hemodiálisis. Hospital Rafael Ángel Calderón Guardia. Revista Clínica de la Escuela de Medicina UCR -HSJD. 3 (IX). Recuperado de http://www.kerwa.ucr.ac.cr/ bitstream/handle/10669/14822/13207-22140-1-SM. pdf? sequence $=1$ \&isAllowed $=\mathrm{y}$

Martínez, M., Martínez, E., \& Andrés, E. (2007). ¿Por qué no diagnosticamos los niveles de depresión, ansiedad y estrés en los pacientes en hemodiálisis? Servicio Psicología - Servicio Nefrología -Fundación Puigvert. Congreso Internacional de Nefrología por internet CINET. 2007; Recuperado de http://cin2007.uninet.edu/en/trabajos/fulltext/95.pdf

Orellana, M. \& Munguía, A. (2008). Insuficiencia Renal y Depresión. Revista de Post grado de Psiquiatría UNAH. 1(3); Recuperado de http://www.bvs.hn/RHPP/pdf/2008/pdf/ Vol1-3-2008-5.pdf

Ortega, E. \& Pérez I. (2004). Intervención sistémica en crisis inesperada en familias donde un miembro es diagnosticado de insuficiencia renal crónica terminal y enfrenta la necesidad de Hemodiálisis. Libro de resúmenes del IX Congreso Peruano de Nefrología y II Curso Iberoamericano de Nefrología, diálisis y trasplante.

Páez, A, Jofrè, M., Marcos, J, Azpiroz, C., \& De Bortoli, M. (2008). Ansiedad y depresión en pacientes con insuficiencia renal crónica en tratamiento de diálisis. Univ.Psychology; 8(1), 117-124. Recuperado de http://web.a.ebscohost.com/ ehost/pdfviewer/pdfviewer?sid=4735f906-6127-4aea-afe9bbf017cbb97a\%40sessionmgr4003\&vid $=5 \&$ hid $=4112$

Perales-Montilla, C., García-León, A., \& Reyes del Paso, G. (2012). Predictores psicosociales de la calidad de vida en pacientes con insuficiencia renal crónica en tratamiento de hemodiálisis. Revista de Nefrología. Órgano Oficial de la Sociedad Española de Nefrología; 32(5): 622-30. Recuperado de http://eds.a.ebscohost.com/ehost/pdfviewer/ pdfviewer?sid=7bfe 89a2-1dfd-413b-8115-74fb26c8bfbc\% 40sessionmgr4001\& vid $=42 \&$ hid $=4205$

Perales-Montilla, C., Duschek, S., \& Reyes-del Paso, G. (2013). Influencia de los factores emocionales sobre el informe de síntomas somáticos en pacientes en hemodiálisis crónica: relevancia de la ansiedad. Revista de Nefrología; 33(6):81625 Recuperado de http://www.revistanefrologia.com

Pérez-Domínguez, T., Rodríguez-Pérez, A., García-Bello, M., Buset-Ríos, N., Rodríguez-Esparragón, F., Parodis-López, Y., \& Rodríguez-Pérez, J. (2012). Progresión de la enfermedad renal crónica. Prevalencia de ansiedad y depresión en la poliquistosis renal autosómica dominante. Revista de Nefrología; 32(3). Recuperado de http://scielo.isciii.es/scielo. php?pid=S0211-69952012000500019\&script=sci arttext

Rubio, R., Sánchez, N., Jiménez, M., Kanahan, D., \& Oria, C. (2012). Comparación de los niveles de depresión, ansiedad y estrés en Pacientes nefrópatas sometidos a diálisis peritoneal y hemodiálisis y su relación con el nivel socioeconómico. Ciudad hospitalaria Enrique tejera (chet) y unidades de diálisis extrahospitalarias. Valencia, estado Carabobo. 2010-2011. Revista 1; (2), 14-21. Recuperado de http://servicio.bc.uc.edu.ve/fcs/avances/volln2/art3.pdf 